\title{
Headache associated with moyamoya disease: a case story and literature review
}

\author{
Victor Zach • David Bezov • Richard B. Lipton • \\ Sait Ashina
}

Received: 11 November 2009/Accepted: 25 November 2009/Published online: 11 December 2009

(C) Springer-Verlag 2009

\begin{abstract}
Headache associated with moyamoya disease (HAMD) is common in moyamoya disease. However, the characteristics and classification of HAMD are largely unknown. We present a case of a 39-year-old woman with HAMD. To characterize and classify the features of this syndrome, the patient was asked to complete a 4-month diagnostic headache diary. There was a total of 15 ictal days. All episodes were without aura. The headache was more commonly pressing (10/15), mild to moderate in severity (14/15), unchanged by physical activity (11/15), and associated with photophobia (10/15). The International Headache Society Classification was utilized to determine that eight episodes met criteria for probable migraine without aura, while seven episodes met criteria for probable frequent episodic tension-type headache. We identified four other case reports of HAMD with partial descriptions of the characteristics. When combined with our patient, the median age was 34 years old (range 6-49, SD 16). Four were female, while the patient with cluster headache was
\end{abstract}

\section{Zach $(\bowtie)$}

Department of Neurology, The Mount Sinai School of Medicine,

The Mount Sinai Hospital, 1 Gustave L. Levy Place,

New York, NY, USA

e-mail: stopstroke@gmail.com

D. Bezov $\cdot$ R. B. Lipton $\cdot$ S. Ashina

Department of Neurology, Montefiore Medical Center,

Albert Einstein College of Medicine, Bronx, NY, USA

\section{R. B. Lipton}

Department of Epidemiology and Population Health, Montefiore Medical Center, Albert Einstein College of Medicine, Bronx, NY, USA

R. B. Lipton · S. Ashina

Montefiore Headache Center, Montefiore Medical Center, Albert Einstein College of Medicine, Bronx, NY, USA male. The median time from headache onset to diagnosis with moyamoya disease was 9.5 months (range $0-192$, SD 88.0). Headaches were described as migraine with aura in two of five cases, hemiplegic migraine in one of five, and cluster headache in one of five. The highest intensity was described as severe in three of three cases, in which headache intensity was reported. Meanwhile, nausea, vomiting, and photophobia were present in two of three cases, where these features were reported, while nausea without vomiting was seen in one of three cases. In all five cases, patients had other neurological symptoms, such as paresis, seizures, visual disturbances, dysarthria, allodynia, ptosis, and unilateral restless leg syndrome. In conclusion, HAMD can present as migraine without aura. It can be the first presenting symptom of moyamoya disease. The headache features are not diagnostic; hence, early neurovascular imaging should be considered in patients with new onset, refractory migraine-like headache, especially in the setting of other neurological symptoms to exclude underlying moyamoya disease. Further reports using headache diaries are needed to better characterize HAMD as well as to determine whether headache with tension-type features is also part of this condition.

Keywords Moyamoya $\cdot$ Headache $\cdot$ Migraine Cluster · Diagnosis

\section{Introduction}

Moyamoya disease is an uncommon illness of cerebral vasculature that is identified by stenosis of the distal internal carotid arteries and their branches. Dilation of small collateral vessels in an attempt to compensate for the decreased blood flow in the anterior circulation produces 
Fig. 1 Conventional cerebral angiogram of our patient. a The LICA is occluded just after the bifurcation of the LCCA, $\mathbf{b}$ the RICA narrows and becomes occluded intracranially (white, dotted lines). Distally, the dilated lenticulostriate arteries create a "puff of smoke" appearance. LICA left internal carotid artery, $L C C A$ left common carotid artery, RICA right internal carotid artery
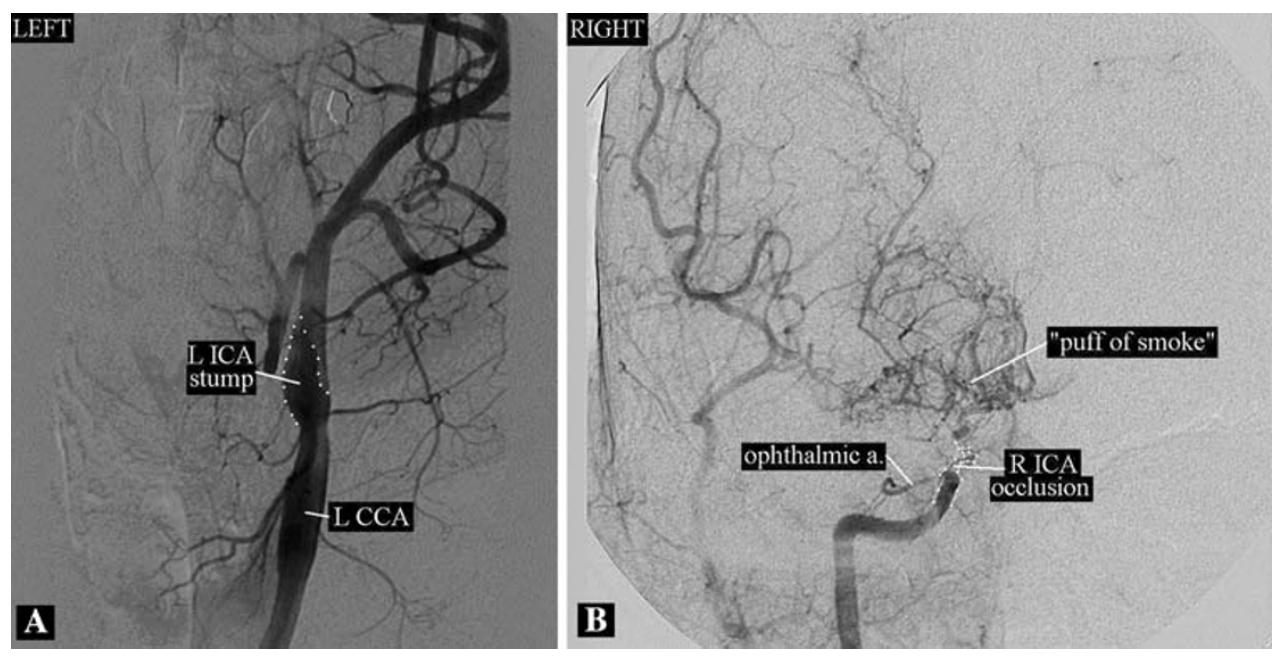

the characteristic "puff of smoke" appearance on angiography [1]. There are primary forms of moyamoya (i.e. moyamoya disease) and forms secondary to radiation to head and neck, Down's syndrome, sickle cell disease, neurofibromatosis type I, severe intracranial atherosclerosis, fibromuscular dysplasia, hyperthyroidism, bacterial meningitis, combined cigarette smoking and oral contraceptive use, Sjogren syndrome and cocaine abuse (i.e. moyamoya syndrome) [1]. In this paper, we will focus on moyamoya disease. Clinically, this illness may result in various combinations of ischemic and hemorrhagic stroke, transient focal neurologic deficits, and seizures. Headache associated with moyamoya disease (HAMD) is common in moyamoya disease and persists in $63 \%$ of patients even after successful surgical revascularization [2]. In some instances, headache may be the presenting symptom of moyamoya disease [2]. However, there are only a few reports of HAMD with a description of headache quality and associated symptoms. We asked a 39-year-old woman with HAMD to keep a headache diary [3] to better characterize her headache. To our knowledge, this is the first time a headache diary was used to characterize HAMD in a case report. We also conducted a literature review and were able to identify three cases of HAMD with description of headache quality and associated symptoms [4-6]. We combined our case with those from the literature review to characterize the features of HAMD. We also discuss the possible pathophysiology of HAMD.

\section{Patient history}

A 39-year-old, right handed, obese, Hispanic woman presented to the neurology clinic at the Montefiore Medical Center with a complaint of a hissing, buzzing noise in her ear for the past 5 months. The noise was daily, continuous, and more pronounced on the left. The patient initially did not report headache, neurological deficits, ear pain, fevers, or rash. She had a history of poorly controlled type 2 diabetes mellitus, hyperlipidemia, hypertension, and asthma. She had no history of neck trauma, chiropractor visits, hyperextension (like in yoga class or in hair salons when they wash hair), nor any motor vehicle accident with whiplash injury. Her medications included aspirin, zolpidem, metformin, fosinopril, atorvastatin, and pioglitazone. Her family history was significant for "minor strokes" in her father and headaches in her brother that could not be further characterized. She was referred to otolaryngology for tinnitus. There she had a normal examination. This led to a referral to neurology. On physical examination, she had bilateral carotid bruits. Otherwise, her neurological examination was normal. A CT scan of the head did not reveal any abnormalities. In addition, she had a CT angiogram of the head and neck, which revealed an occlusion of both internal carotid arteries and reconstitution of the distal middle cerebral arteries via collaterals. The left internal carotid artery (ICA) smoothly tapered just distal to the common carotid bifurcation, while the right ICA tapered more distally. A conventional catheter cerebral angiogram confirmed the occlusion of the left ICA within the neck and the occlusion of the right ICA just above the ophthalmic artery origin. Collateral blood supply to the anterior circulation from the external carotid arteries as well as from the posterior circulation was demonstrated. Furthermore, there were dilated, tortuous lenticulostriate, and thalamostriate arteries with a typical, "puff of smoke" appearance (Fig. 1).

Evaluation for contributing hypercoagulable states included a C-reactive protein, a vasculitis work-up (ANA, ANCA, RF, anti-Ro, and anti-La), an RPR and a sickle cell screen. These tests were unrevealing. The patient had no history of cocaine or illicit drug use. She did not have 
Down's syndrome. There was no evidence of severe atherosclerosis, fibromuscular dysplasia, neurofibromatosis, or craniopharyngioma. On angiography, there was no evidence of dissection. In contrast, the dilated collaterals were present bilaterally and both of the ICAs were occluded. The intracranial location of the right ICA occlusion is consistent with moyamoya disease. The left ICA tapered just past the bifurcation of the common carotid, which is a finding that may be seen with atherosclerosis $[7,8]$ or moyamoya disease. There was no other evidence of atherosclerotic disease in the remaining cerebral vasculature. Based on these findings, we diagnosed the patient with moyamoya disease.

At 2 months follow-up, the patient complained of episodic headaches that were bilateral, frontal, pressure-like, moderate to severe in intensity, lasting for $3 \mathrm{~h}$, associated with photophobia, allodynia, and light headedness. Following the consultation, the patient was instructed to complete a 4-month diagnostic headache diary. She had a headache on 15 out of 121 days (12\% ictal, $88 \%$ headache free). Her headaches were treated with naproxen and acetaminophen. The patient is being followed at the neurology clinic. She is currently not scheduled for any bypass surgery or revascularization.

\section{Results and literature review}

We used a diagnostic headache diary and the second edition of the International Classification of Headache Disorders (ICHD-II) from the International Headache Society (IHS) to classify our patient's headache [9]. The headache features derived from the diary are presented in Table 1 . The ICHD-II [9] of our patient's headache is presented in Table 2. Prior literature describes HAMD as migrainous in quality and refractory to both prophylactic and abortive medical therapies [2]. Recently, cluster headache was reported in moyamoya disease [10]. Surprisingly, using the ICHD-II, we found that our patient's HAMD was not always migrainous. Rather, it was an almost even mixture of probable migraine without aura and tension-type headache. Uncharacteristically, our patient responded to abortive agents and did not require prophylactic therapy.

In addition to our case, we identified four [4-6, 10] other case reports with a detailed description of the headache pattern in moyamoya for a total of five cases. The median age of our five patients with HAMD was 34 years old (range 6-49, SD 16.0). Four were female while the patient with cluster headache was male. The median time from headache onset to diagnosis with moyamoya disease was 9.5 months (range 0-192, SD 88.0). Headaches were described as migraine with aura in two of five cases, hemiplegic migraine in one of five, and cluster headache in
Table 1 Percentages of headache characteristics in a patient with moyamoya disease during 4-month period $(n=15)$

\begin{tabular}{|c|c|}
\hline Characteristics based on 15 episodes & $n(\%)$ \\
\hline \multicolumn{2}{|l|}{ Aura } \\
\hline None & $15(100)$ \\
\hline \multicolumn{2}{|l|}{ Localization } \\
\hline Unilateral & $8(53)$ \\
\hline Bilateral & $7(47)$ \\
\hline \multicolumn{2}{|l|}{ Quality } \\
\hline Pressing & $10(67)$ \\
\hline Pulsating & $4(27)$ \\
\hline Throbbing & $1(7)$ \\
\hline \multicolumn{2}{|l|}{ Severity } \\
\hline Mild & $7(47)$ \\
\hline Moderate & $7(47)$ \\
\hline Severe & $1(7)$ \\
\hline \multicolumn{2}{|l|}{ Effect of physical activity } \\
\hline Worse & $4(27)$ \\
\hline Unchanged & $11(73)$ \\
\hline Better & $0(0)$ \\
\hline \multicolumn{2}{|l|}{ Nausea } \\
\hline None & $12(80)$ \\
\hline Mild & $2(13)$ \\
\hline Moderate & $0(0)$ \\
\hline Severe & $1(7)$ \\
\hline \multicolumn{2}{|l|}{ Photophobia } \\
\hline None & $5(33)$ \\
\hline Mild & $6(40)$ \\
\hline Moderate & $3(20)$ \\
\hline Severe & $1(7)$ \\
\hline \multicolumn{2}{|l|}{ Phonophobia } \\
\hline None & $8(53)$ \\
\hline Mild & $6(40)$ \\
\hline Moderate & $1(7)$ \\
\hline Severe & $0(0)$ \\
\hline \multicolumn{2}{|l|}{ Provocative factors } \\
\hline None & $11(73)$ \\
\hline Stress & $2(13)$ \\
\hline Work & $1(7)$ \\
\hline Sleep & $1(7)$ \\
\hline
\end{tabular}

Table 2 IHS Classification of Headache Types in our patient with moyamoya disease

\begin{tabular}{ll}
\hline IHS Classification ICHD-II $(n=15)$ & $n(\%)$ \\
\hline Probable migraine without aura 1.6.1. & $8(53)$ \\
Probable frequent episodic tension-type headache 2.4.2. & $7(47)$ \\
\hline
\end{tabular}

one of five. The highest intensity was described as severe in three of three cases, in which headache intensity was reported. Meanwhile, nausea, vomiting and photophobia 
were present in two of three cases where these features were reported, while nausea without vomiting was seen in one of three cases. In all five cases, patients had other neurological symptoms, such as paresis, seizures, visual disturbances, dysarthria, allodynia, ptosis, and unilateral restless leg syndrome.

\section{Discussion}

The mechanism of HAMD is uncertain and may be multifactorial. Moyamoya disease results in narrowing and ultimately occlusion of intracranial vasculature. It is possible that HAMD is also caused by this blood vessel pathology [2]. The role of cerebral artery nociceptors in generation of HAMD is supported by studies where stretch of intracerebral vasculature produced nausea and referred pain in regions typically involved in migraine attacks $[11,12]$.

Furthermore, cerebral aneurysms and arteriovenous malformations are frequently found in moyamoya disease (incidence 11-14.2\%) and may account for the headache in at least a subset of patients by two possible mechanisms [2, $13,14]$. One mechanism may be the stimulation of trigeminal nociceptors due to stretch caused by the enlarging aneurysm [15]. The second mechanism may be a sentinel aneurysmal bleed into the subarachnoid space, which has previously been associated with the generation of headache with pulsating quality accompanied by photophobia, nausea, and vomiting [15].

Neurogenic inflammation seen in moyamoya disease may also play a role in generating HAMD. The inflammation results in stretch of perivascular nociceptors innervating intracranial vasculature and leads to a release of pro-nociceptive mediators. These include substance $\mathrm{P}$ and calcitonin gene-related peptide from trigeminal nerve endings. The release leads to activation of nociceptors producing both generation and maintenance of the headache $[11,16]$.

Finally, the progressive intracranial stenosis seen in moyamoya disease leads to cerebral hypoperfusion and induces an intracranial oligemic state. This hypoperfusion has been described as an etiology of headache as well [5]. Indeed, some patients experience an improvement in HAMD after surgical intervention intended to improve cerebral blood flow $[2,17]$. This notion is further supported by the fact that hyperventilation, which is known to cause cerebral vasoconstriction and thus hypoperfusion, has been associated with both headache generation and transient ischemic attacks in moyamoya disease [2].

In conclusion, HAMD can present as migraine without aura. It can be the first presenting symptom of moyamoya disease. The headache features are not diagnostic, hence early neurovascular imaging should be considered in patients with new onset, refractory migraine-like headache, especially in the setting of other neurological symptoms to exclude underlying moyamoya disease. Further reports using headache diaries are needed to better characterize HAMD as well as to determine if headache with tensiontype features is also part of this condition.

Acknowledgments The patient kindly gave verbal consent for the publication of the case. We would like to thank Dr. Li-fen Chen for the participation in the care of this patient. We would like to thank Dr. Tamara Zach for reviewing the manuscript.

Conflict of interest None.

\section{References}

1. Scott RM, Smith ER (2009) Moyamoya disease and moyamoya syndrome. N Engl J Med 360(12):37-1226

2. Seol HJ, Wang KC, Kim SK, Hwang YS, Kim KJ, Cho BK (2005) Headache in pediatric moyamoya disease: review of 204 consecutive cases. J Neurosurg 103(Suppl):439-442

3. Russell MB, Rasmussen BK, Brennum J, Iversen HK, Jensen RA, Olesen J (1992) Presentation of a new instrument: the diagnostic headache diary. Cephalalgia 12(6):369-374

4. Bernstein AL (1993) Hemiplegic migraine and moyamoya disease (letter). Am J Dis Child 147:718-719

5. Park-Matsumoto YC, Tazawa T, Shimizu J (1999) Migraine with aura-like headache associated with moyamoya disease. Acta Neurol Scand 100:119-121

6. Liu X-F, Jung DK (1999) Moyamoya disease and migraine-like headaches. Schweiz Arch Neurol Psychiatr 150:272-274

7. Gorelick PB, Caplan LR, Hier DB, Parker SL, Patel D (1984) Racial differences in the distribution of anterior circulation occlusive disease. Neurology 34:54-59

8. Caplan LR, Gorelick PB, Hier DB (1986) Race, sex, and occlusive cerebrovascular disease: a review. Stroke 17:648-655

9. Headache Classification Subcommittee of the International Headache Society (2004) The International Classification of Headache Disorders: 2nd edition. Cephalalgia 24(Suppl 1):9-160

10. Sewell RA, Johnson DM, Fellows DW (2009) Cluster headache associated with moyamoya. J Headache Pain 10:65-67

11. Olesen J, Burstein R, Ashina M, Tfelt-Hansen P (2009) Origin of pain in migraine: evidence for peripheral sensitization. Lancet Neurol 8:679-690

12. Ray BS, Wolff HG (1940) Experimental studies on headache: pain sensitive structures of the head and their significance in headache. Arch Surg 41:813-856

13. Oi S, Yamada H, Sasaki K, Matsumoto H (1986) Incidence and characteristics of cerebrovascular disorders in children-critical analysis of 120 cases experienced at a children's general hospital. No Shinkei Geka 14(2):161-168

14. Kawaguchi S, Sasaki T, Morimoto T, Kakizaki T, Kamada K (1996) Characteristics of intracranial aneurysms associated with moyamoya disease: a review of 111 cases. Acta Neurochir (Wien) 138:1287-1294

15. De Falco FA (2004) Sentinel headache. Neurol Sci 25(Suppl 3):S215-S217

16. Panconesi A, Bartolozzi ML, Guidi L (2009) Migraine pain: reflections against vasodilation. J Headache Pain 10(5):317-325

17. Matsushima Y, Aoyagi M, Niimi Y, Masaoka H, Ohno K (1990) Symptoms and their pattern of progression in childhood moyamoya disease. Brain Dev 12:784-789 